the initial invasive staging modality after bronchoscopy plus TBNA.

Since the publication of our editorial comment, two further prospective studies have investigated the addition of EUS-FNA to a standard work-up for unselected LC patients $[3,4]$. In one of these studies, the results of EUS-FNA were blinded [3]. The conclusions were clear; EUS-FNA, when added to MS (and TBNA), improves the pre-operative staging of $\mathrm{LC}$, resulting in a reduced rate of futile thoracotomies.

How much additional proof do we need? Should we ask for more evidence from new less invasive methods that exists for the standard methods? When is the right time to change or add to standards? We believe, based on the current literature, that the time has come for EUS-FNA.

However, the aim of our editorial comment was not to claim the superiority of one method above the other, but to inform thoracic specialists that, according to the available evidence, the addition of endoscopic ultrasound-guided fine-needle aspiration to a standard work-up improves selection of surgically curable patients with lung cancer.
P. Vilmann and S.S. Larsen

Gentofte University Hospital, Hellerup, Denmark.

\section{REFERENCES}

1 Vilmann P, Larsen SS. Endoscopic ultrasound-guided biopsy in the chest: little to lose, much to gain. Eur Respir J 2005; 25: 400-401.

2 Larsen SS, Vilman P, Krasnik M, et al. Endoscopic ultrasound-guided biopsy versus mediastinoscopy for analysis of paratracheal and subcarinal lymph nodes in lung cancer staging. Lung Cancer 2005; 48: 85-92.

3 Annema JT, Versteegh MI, Veselic M, et al. Endoscopic ultrasound added to mediastinoscopy for preoperative staging of patients with lung cancer. JAMA 2005; 294: 931-936.

4 Larsen SS, Vilman P, Krasnik M, et al. Endoscopic ultrasound guided biopsy performed routinely in lung cancer staging spares futile thoracotomies: preliminary results from a randomised clinical trial. Lung Cancer 2005; 49: 377-385.

DOI: 10.1183/09031936.06.00107705

\title{
Projections of COPD in males in the Netherlands
}

\section{To the Editors:}

In a recent issue of the European Respiratory Journal, projections for a large increase in chronic obstructive pulmonary disease (COPD) in females in the Netherlands up to 2025 were recorded in the study by HOOGENDOORN et al. [1]. These are depressing, but expected given recent observed trends and the modest changes in smoking made by Dutch females since 1980 $[2,3]$. However, males in the Netherlands, who had the highest known usage of tobacco products per adult of any country from the 1930s to the 1950s [2], have considerably reduced their cigarette consumption since the 1970s [2, 3]. Therefore, it is disconcerting to see that continuing upward trends in COPD, albeit smaller in scale, are also projected for Dutch males. Where the predicted time trend is shown [1], there is little, if any, slowing of the rate of increase over the whole period up to 2025. In contrast, according to the World Health Organization (WHO) compilation of national statistics presented by PETO et al. [4], total deaths from lung cancer have recently begun to fall in males within the European Union. In the Netherlands, total male deaths from lung cancer and rates per 100,000 in both the total adult population, and those aged 75-79 yrs, all declined progressively between 1985 and 2000. As discussed in the accompanying editorial by MANNINO [5], and elsewhere [6], slower and less dramatic benefits would be expected in COPD than in lung cancer after quitting smoking. Nevertheless, WHO data on COPD mortality in the Netherlands [4] also indicate that between 1990 and 2000 total male deaths have been stable, whereas death rates per 100,000 for the total adult population, and for males aged 75-79 yrs, have both declined by a similar proportionate amount.
While recognising that the primary purpose of the authors' model is to estimate total future costs due to chronic obstructive pulmonary disease, regardless of how these costs arise, the authors hardly comment on the extent to which their estimates depend on demographic changes, in particular, increased age of the population (which perhaps represents medical "success" but increases costs and worries pension funds) and how much depends on the persistence of smokingrelated disease (which is amenable to preventive medicine). The increases presented for prevalence and mortality could potentially be explained entirely by population ageing, but there is no quantification of these two major factors to support or refute this possibility. Such information might help the medical reader to better understand the paradox that, while smoking is the most important aetiological factor for causing physician-diagnosed chronic obstructive pulmonary disease, its prevalence and mortality can be projected to continue to increase even after 45 yrs of reduction in cigarette smoking by Dutch males.

\section{N.B. Pride}

Dept of Thoracic Medicine, Imperial College, London, UK

\section{REFERENCES}

1 Hoogendoorn M, Rutten-van Molken MPMH, Hoogenveen $\mathrm{RT}$, et al. A dynamic population model of disease progression in COPD. Eur Respir J 2005; 26: 223-233.

2 Forey B, Hamling J, Lee P, Wald N. International Smoking Statistics. A Collection of Historical Data from 30 Economically Developed Countries. 2nd Edn. Wolfson 
Institute of Preventive Medicine, London and Oxford, Oxford University Press, 2002.

3 Loddenkemper R, ed. European Lung White Book. Huddersfield, European Respiratory Society, 2003.

4 Peto R, Lopez AD, Boreham J, Thun M, Heath C Jr. Mortality from Smoking in Developed Countries 1950-2000. 2nd Edn. Oxford, Oxford University Press, 2004.

5 Mannino D. Chronic obstructive pulmonary disease in 2025: where are we headed? Eur Respir J 2005; 26: 189.

6 Pride NB. Smoking cessation: effects on symptoms, spirometry and future trends in COPD. Thorax 2001; 56: Suppl. 2, 7-10.

DOI: $10.1183 / 09031936.06 .00107605$

\section{From the authors:}

We would like to thank N.B. Pride for the interest in our article [1] and the opportunity to elaborate on our projections of the increasing prevalence of chronic obstructive pulmonary disease (COPD) in the Dutch population.

First, we would like to emphasise that, like all model projections, the numbers should not be considered as infallible projections for the future. This was not the aim of our model. Rather it has been developed as a tool to help explain the current situation, given current COPD treatment and knowledge on the demographics and smoking trends, and to support policy makers in their choices. While the current practice scenario may be interesting on its own, the main purpose of model projections over a certain time period is to enable comparison of different policy choices regarding prevention, as with the smoking cessation measures used as an illustration in our article.

N.B. Pride addressed the issue as to why the prevalence of COPD for males increased despite the decline in smoking prevalence over the last 30 yrs. In our current practice scenario, the prevalence of COPD was projected to increase from 24 to 33 per 1,000 inhabitants of all ages for males and from 15 to 27 per 1,000 inhabitants for females.

Indeed, the smoking prevalence in Dutch males has decreased substantially since 1970 . As a consequence, the percentage of ex-smokers in the Dutch population increased during this period. The relative risk (RR) of these ex-smokers for developing COPD is still increased (males aged 45-69 yrs, $\mathrm{RR}=11.2$; males aged $>70 \mathrm{yrs}, \mathrm{RR}=7.4$ ). Many of these exsmokers have reached the older age classes, where the incidence of COPD is high. The RR to develop COPD in our model is based on the average RR among ex-smokers. This group of ex-smokers consists of males who recently gave up smoking and males who gave up smoking a while ago. On average, this RR represents the entire group of ex-smokers well, unless there is a relatively large proportion of ex-smokers who gave up smoking a long time ago. In the latter case, the RR may be overestimated. However, there is evidence that the RR of developing COPD after smoking cessation does not decline as fast as it does for other smoking-related diseases, such as lung cancer [2].
Our dynamic life-table model accounts for demographic changes, as well as changes in smoking prevalence. The past smoking behaviour of the population is present in the current distribution of nonsmokers, smokers and ex-smokers in the different age and sex classes. This distribution changes each year as a result of ageing of the birth cohorts and the application of age- and sex-specific start, stop and restart rates [1]. Commonly, projections of the burden of disease are not based on dynamic life-table models, but on simple demographic projections in which age- and sex-specific prevalence rates are multiplied by the expected number of people in the age and sex classes in the future (Statistics Netherlands, Voorburg/Heerlen, the Netherlands). We recently compared the results of our model with a simple demographic projection [3]. Figure 1 shows the results of this comparison for the Netherlands. For males, the simple demographic projection resulted in a considerably higher number of patients than our model projection had predicted. Accounting for changes in smoking prevalence decreases the projection for the total number of male COPD patients for the year 2025 from 315,000 to 270,000 . For females, the opposite is seen. Hence, not taking into account changes in smoking prevalence would certainly overestimate the burden of COPD among males. These findings are in accordance with an earlier publication [4].

Figure 2 also sheds more light on the issue raised by N.B. Pride. It shows the age specific prevalence rates of COPD among males. For all age classes except $80-84$ yrs and $>85$ yrs, the COPD prevalence rate over the period 2000-2025 decreased. As the number of people in the older age classes in the general population increased considerably, the absolute number of male COPD patients increased from 188,000 to 270,000 , despite a decline in age-specific prevalence rates. Thus, as the total number of males in the population hardly changed (from 7.8 to 8.2 million), the prevalence rate for males expressed per 1,000 inhabitants increased from 24 to 33 .

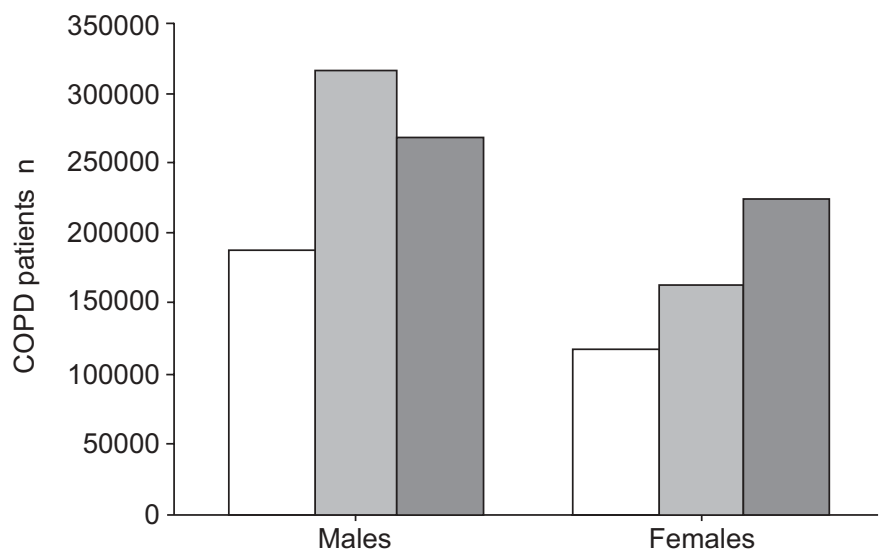

FIGURE 1. Absolute number of chronic obstructive pulmonary disease (COPD) patients in $2000(\square)$, simple demographic projections for the year 2025

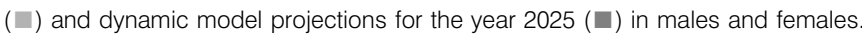

